"This is the peer reviewed version of the following article: [Energy Technology, 2018, 6 (7), pp. 1352 - 1360], which has been published in final form at

[https://onlinelibrary.wiley.com/doi/abs/10.1002/ente.201700680]This article may be used for non-commercial purposes in accordance with Wiley Terms and Conditions for Self-Archiving 


\section{State-of-Power Estimation of Li-ion Battery Considering Battery Surface Temperature}

Xintian $\mathrm{Liu}^{1}$, Yao $\mathrm{He}^{1}$, Guojian Zeng ${ }^{1}$, Jiangfeng Zhang ${ }^{2}$, Xinxin Zheng ${ }^{1}$

1. New Energy Automotive Engineering Research Institute, Hefei University of Technology, Hefei, 230009, China

2. School of Electrical, Mechanical and Mechatronic Systems, University of Technology Sydney, Australia

8

Abstract: The State-of-Power (SOP) of Li-ion battery (LIB) is an important evaluation index for security control and energy recovery of electric vehicles. Major state estimation methods are only applicable to fixed room temperature at $20^{\circ} \mathrm{C}$. Actually, the battery capacity and resistance vary dramatically with the change of battery surface temperature in the working process of the battery, which causes significant errors in state estimation if only room temperature is assumed. The inaccurate state may cause further excessive current at high or low temperatures to affect security and life cycle of the battery. Therefore, a novel state estimation method applicable to various battery surface temperatures is developed in this paper. This method establishes Capacity-Temperature relations and Resistance-Temperature relations from experimental data to predict more accurately battery capacity and resistance in the full temperature range, then applies the Extend Kalman Filter technique to estimate the State of Charge, and an algorithm with multi-parameters constrained to estimate the SOP of LIB. Simulation and experimental results show that the 
proposed method can obtain accurate SOP results at different temperatures.

Keywords: Li-ion battery; State-of-Power; Temperature model; Temperature rising characteristic; algorithm with multi-parameters constrained

\section{Introduction}

In recent years, electric vehicle $(\mathrm{EV})$ technology has been developed quickly because of increasingly serious energy supply shortage and environmental concerns. As the main power source of EVs, Li-ion battery (LIB) has attracted intensive research. State-of-Power (SOP) is an important evaluation index to characterize the charging or discharging peak power of LIB. SOP is used to evaluate whether the battery pack has sufficient power to meet the starting or acceleration demand of EVs. At the braking state, SOP can help to determine the maximum energy recovered by the battery and thus avoid overcharging. Moreover, accurate SOP estimation result can benefit vehicle performance control by optimizing battery capacity and size $[1,2,3]$. Therefore, it is important to estimate SOP accurately, and this paper aims to develop a new SOP estimation method which will have a satisfactory accuracy under various temperatures.

SOP of LIB is related to battery capacity, resistance, State-of-Charge (SOC) and ambient temperature amongst others [4], and the estimation of SOP needs to consider all these factors. In Ref. [5], Hybrid Pulse Power Characterization (HPPC) method is applied to estimate SOP. This method uses internal resistance, open-circuit voltage (OCV) and cut-off voltage to obtain the peak power of the battery. Another SOP estimation method based on SOC limits (MSL) is introduced in Ref. [6], where discharging peak current is obtained by the current SOC and minimum SOC limit over a given time period. In Ref. [1], a SOP estimation method based on voltage limit is proposed, in which 
complex but more accurate battery models, such as an Equivalent Circuit Model (ECM) or Combined Model (CM), are applied to build the state-space equations of LIB. The peak current at the voltage limit is obtained to calculate the SOP of the battery.

In the above methods, the HPPC method and the MSL are unable to describe the characteristics of lithium batteries accurately due to the inaccurate models used, while the method based on voltage limit ignores the SOC limits and the maximum current provided by the manufacturer. Therefore, these three methods often cause the estimated SOP greater than the actual peak power, and the battery controlled by such an over-estimated SOP will suffer from over-charging, over-discharging or over-current and thus a shortened battery life period.

To improve the accuracy of the SOP estimation, some researchers propose the neural network method [7] and Algorithm with Multi-Parameters Constrained (AMPC) [2,3,8,9]. Neural network method is suitable for state estimation of LIB with a high accuracy degree. However, this method relies on a lot of training data and a proper training technique, otherwise its accuracy will be compromised. The AMPC compares the maximum current provided by the manufacturer with the peak currents obtained from two methods: the one based on SOC limit and the one based on voltage limit. Then it chooses the minimum one in order not to exceed the allowed current and power limit.

Thevenin equivalent circuit model is used in AMPC to estimate the SOP in Ref. [8]. The electro-chemical polarization battery model is used in $[2,3,9]$ to estimate the peak current at voltage limits, and then the SOP is estimated by AMPC.

Major battery state estimation methods are always applied at fixed room temperature (FRT) at $20^{\circ} \mathrm{C}$, and the parameters of battery capacity and resistance are set as constants. Some methods are 
63 applied at fix ambient temperature (FAT) which is equal to the initial battery surface temperature,

64 and the parameters of battery capacity and resistance are set as functions varying with the ambient

65 temperature but not the battery surface temperature. However, significant surface temperature

66 change is inevitable in EV batteries because of the heat generated by the battery itself. Actually,

67 these parameters of LIB change against SOC and battery surface temperatures. And particularly the

68 change of battery temperature will cause sharp fluctuations in those parameters. When the battery

69 surface temperature decreases, the slow kinetics of reactions and the reduced ionic conductivity of

70 electrolyte result in lower capacity and higher resistance of battery [10,11]. Battery impedance online

71 estimation and adaptive power prediction are studied considering such a battery surface temperature

72 problem in [12], however, it studies only the temperature at $10^{\circ} \mathrm{C}$.

Targeting at these problems, a state estimation method considering battery dynamic surface temperature is proposed in this paper. The effect of battery temperature on the parameters of LIB is

75 studied. The capacity and resistance of the LIB are tested at different temperature to build the 76 temperature models which can help to identify how the parameters of LIB vary against battery

77 temperature. The battery performance model is optimized with the aid of temperature models to 78 describe the dynamic characteristics of the LIB at different temperatures. The Extend Kalman Filter 79 (EKF) technique at DBT is applied to estimate the battery SOC, and then an AMPC at DBT with the 80 constraints from SOC and other parameters is applied for the estimation of battery SOP which will 81 ensure the safe operation and energy recovery efficiency of the battery. Finally Dynamic Stress Test 82 (DST) [13] is carried out under different temperatures to verify the accuracy of the temperature models and SOP estimation algorithm. Compared with existing methods, this new method achieves 
90

the complete SOP estimation in the full temperature range with high accuracy. Moreover, this method is simple and suitable for practical application.

This paper is organized as follows. Temperature rising characteristic of LIB and temperature models of capacity and resistance are shown in Section 2. An SOP estimation model considering battery temperature is presented in Section 3. The experiments and simulation results are reported in Section 4. The conclusions are presented in Section 5.

\section{Building the dynamic model of LIB}

Since this paper targets to develop a new SOP estimation method applicable to all temperatures, it is necessary to study first how the relevant battery parameters change against temperature. Based on experimental data, this section establishes the relation between temperature, battery resistance and capacity, and then a dynamic model of LIB is built.

\subsection{Temperature characteristics of LIB}

The cylindrical LIB of type IFP3213100EA manufactured by Guoxuan High-Tech Power is chose to study its temperature characteristics, and the rated capacity is 9AH. The LIB is placed in an incubator, and ambient temperature in the incubator is set as $-10^{\circ} \mathrm{C}, 0^{\circ} \mathrm{C}$ and $25^{\circ} \mathrm{C}$ respectively. Then the constant current discharge test of 2C and Dynamic Stress Test (DST) [13] are conducted to observe the temperature rising of the battery, where $2 \mathrm{C}$ represents two times of rated battery current. . The DST curve and temperature rising results are shown in Fig.1, Fig.2 and Fig.3: 


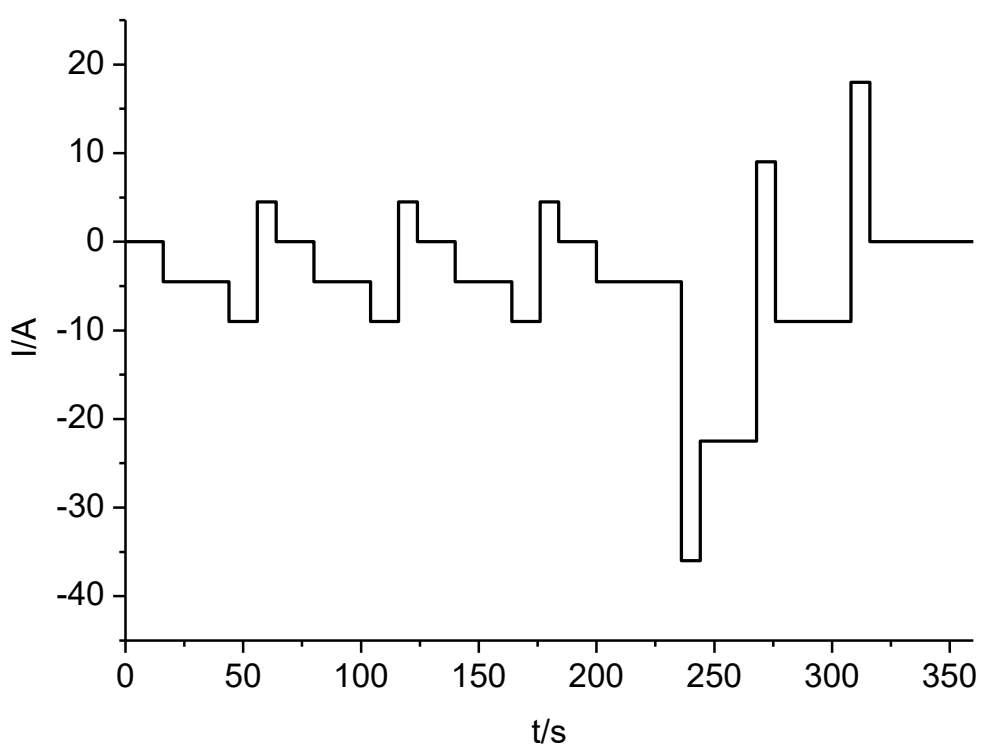

104

Fig. 1 Working condition of a single DST

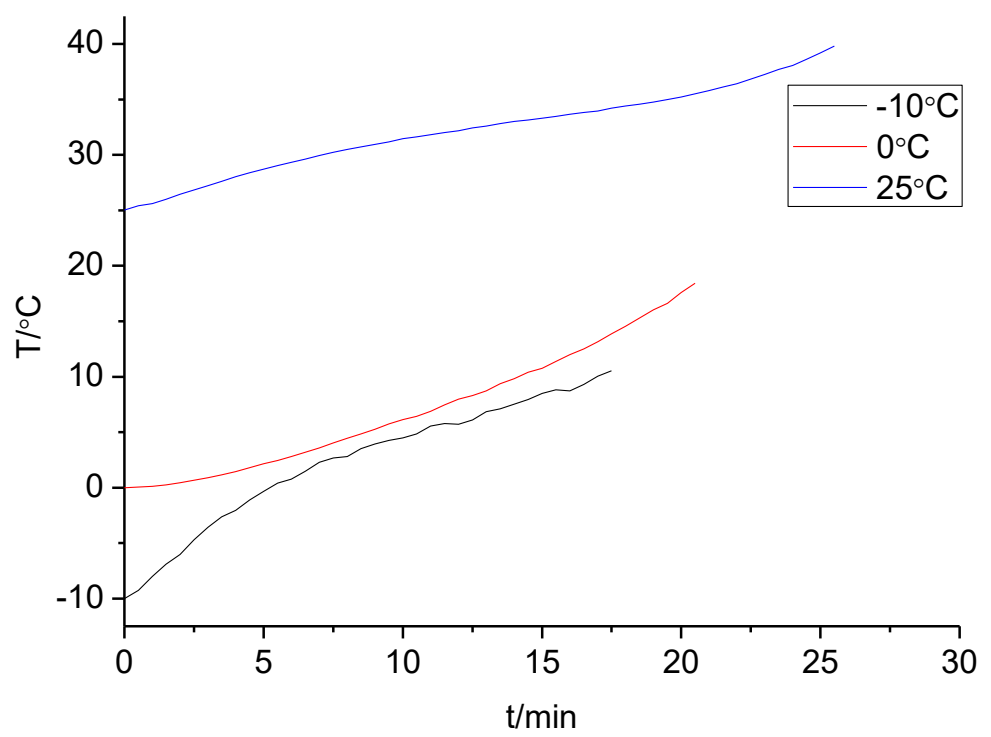

105

Fig.2 Surface temperature rising in constant current discharge test of $2 \mathrm{C}$

106

107 


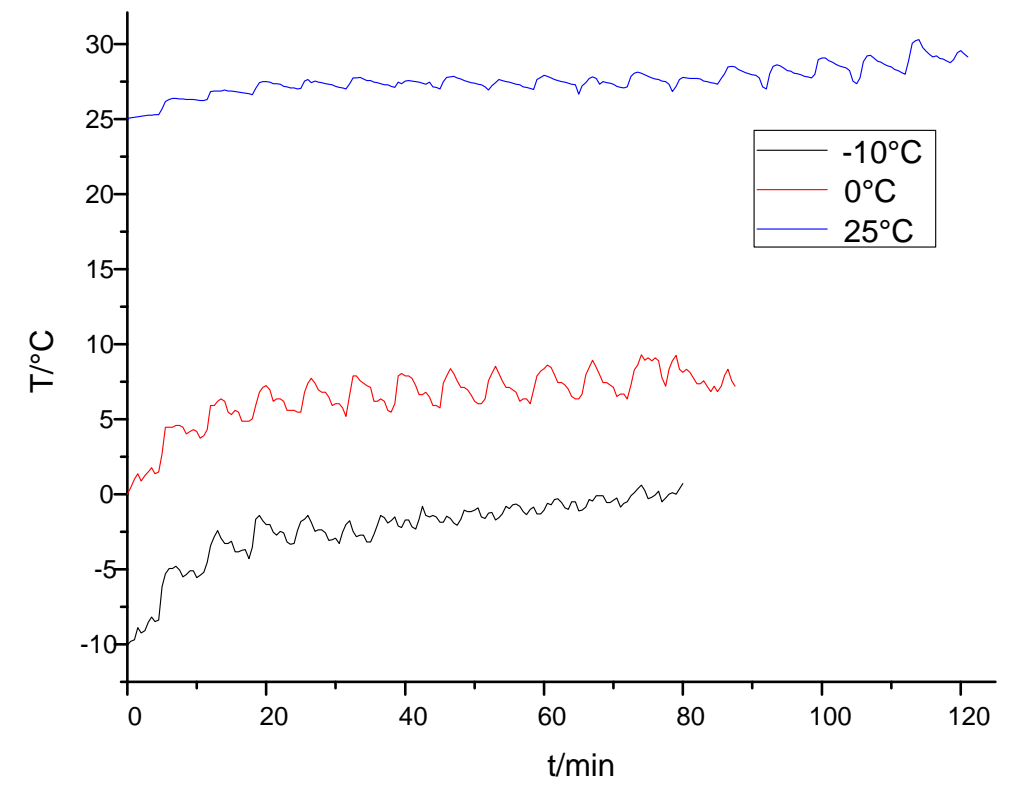

Fig.3 Surface temperature rising of DST

Fig. 1 illustrates the test conditions of a single DST, where the current (in Amperes) at different

111 time instant is given. Fig.2 and Fig.3 show the surface temperature rising of LIB at different ambient

112 temperature in constant current discharge test and DST. And the temperature increases significantly

113 at the discharging process as shown in Table 1:

114

Table 1 Results of Battery Surface Temperature Rising at Different Working Condition

\begin{tabular}{ccccc}
\hline \multirow{2}{*}{$\begin{array}{c}\text { Working } \\
\text { Condition }\end{array}$} & Ambient Temperature & Surface Temperature & Temperature increased & Working Time \\
\hline \multirow{2}{*}{$\begin{array}{c}\text { Constant Current } \\
\text { Test }\end{array}$} & $-10^{\circ} \mathrm{C}$ & $10.55^{\circ} \mathrm{C}$ & $20.55^{\circ} \mathrm{C}$ & 17.5 min \\
\cline { 2 - 5 } & $0^{\circ} \mathrm{C}$ & $18.42^{\circ} \mathrm{C}$ & $18.42^{\circ} \mathrm{C}$ & 20.5 min \\
\cline { 2 - 5 } & $25^{\circ} \mathrm{C}$ & $39.81^{\circ} \mathrm{C}$ & $14.81^{\circ} \mathrm{C}$ & $25.5 \min$ \\
\hline \multirow{2}{*}{ DST } & $-10^{\circ} \mathrm{C}$ & $0.81^{\circ} \mathrm{C}$ & $10.81^{\circ} \mathrm{C}$ & $80 \mathrm{~min}$ \\
\cline { 2 - 5 } & $0^{\circ} \mathrm{C}$ & $8.73^{\circ} \mathrm{C}$ & $8.73^{\circ} \mathrm{C}$ & $91.5 \mathrm{~min}$ \\
\hline & $25^{\circ} \mathrm{C}$ & $30.06^{\circ} \mathrm{C}$ & $5.06^{\circ} \mathrm{C}$ & $121 \mathrm{~min}$ \\
\hline
\end{tabular}

117 The ambient temperature, the battery surface temperature and the temperature increased after 118 the whole discharging process from $100 \%$ SOC to the protection voltage, the continuous working 
119 time are shown in Table 1. Fig.2, Fig.3 and Table 1 show that the temperature rises quickly at low

120 ambient temperatures. If we compare the results at the same time interval, the conclusion is more

121 obvious. The heat at the battery discharge process consists of the heat from resistance and the heat

122 from chemistry reactions, where the heat from resistance occupies the majority of the heat [14]. So

123 the underlying reason of the quick temperature rise is that the resistance is higher at low

124 temperatures, and thus low ambient temperature has a more significant impact on state estimation of

125 LIB.

126

$127 \quad 2.2$ Temperature model of battery capacity

128 In order to find out how the battery usable capacity changes at different temperatures, a fully

129 charged battery is discharged with constant current at different temperatures in an incubator. To

130 avoid the impact of temperature rising on capacity test, the discharging current is set as $0.1 \mathrm{C}$, and

131 the surface temperature is detected in real time to ensure that the battery temperature rises within a

132 permitted range. The ambient temperature is set as $-20^{\circ} \mathrm{C},-10^{\circ} \mathrm{C}, 0^{\circ} \mathrm{C}, 10^{\circ} \mathrm{C}, 20^{\circ} \mathrm{C}, 30^{\circ} \mathrm{C}$ and $40^{\circ} \mathrm{C}$

133 in turn. The measured LIB usable capacities under different temperatures are shown in Fig. 4. 


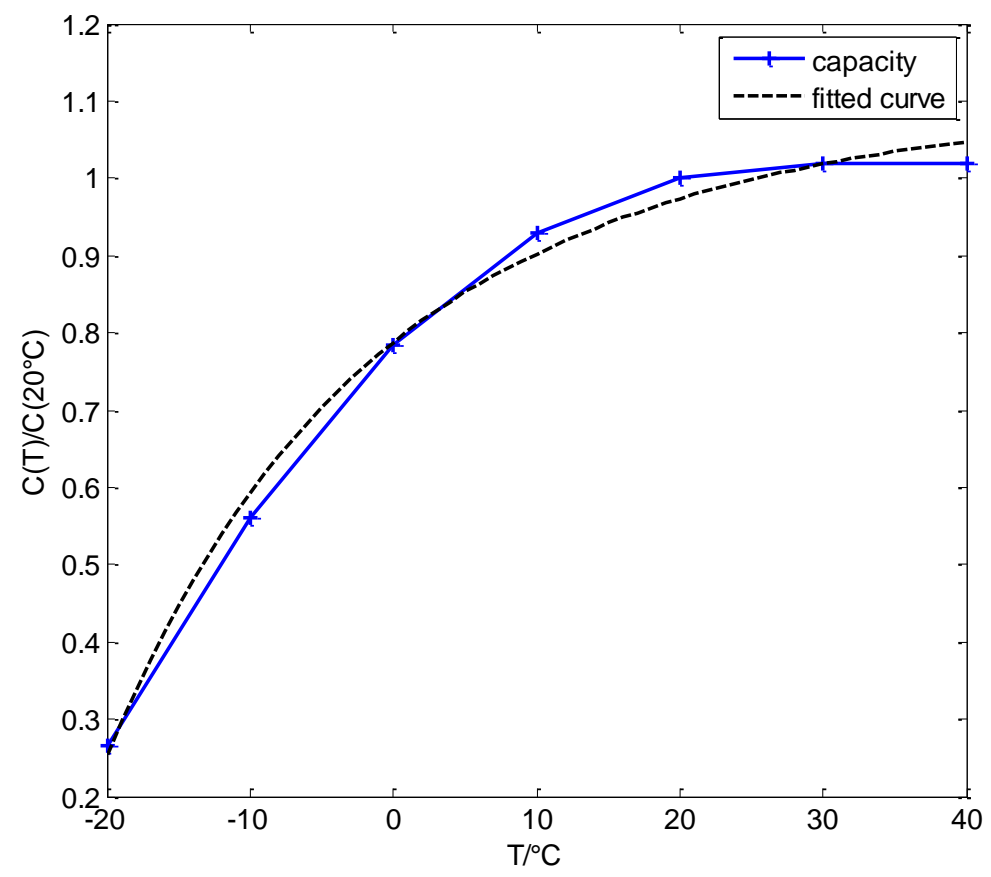

In Fig.4, the vertical axis is the ratio of capacity under the current temperature and usable capacity under room temperature $\left(20^{\circ} \mathrm{C}\right)$, the horizontal axis is the ambient temperature. The figure shows that the LIB usable capacity decreases fast at lower ambient temperatures. At $-20{ }^{\circ} \mathrm{C}$, the capacity is only about $30 \%$ of the rated capacity which is measured at room temperature $\left(20^{\circ} \mathrm{C}\right)$. As

140 the temperature rises, the change rate of the usable capacity gradually reduces. At $40^{\circ} \mathrm{C}$, the capacity 141 is about $105 \%$ of the rated capacity.

143 of temperature on LIB capacity, where $C_{T}$ is the capacity at temperature $T, C_{0}$ is the capacity at

$14420^{\circ} \mathrm{C}$. The curve is fitted by the Arrhenius equation [15], and $\lambda_{C}$ can be expressed as:

$$
\lambda_{C}=C_{T} / C_{0}=B_{C} e^{-\mathrm{E}_{\mathrm{a}}^{\mathrm{c}} / R T}+A_{C}
$$

146 where $B_{C}$ is the pre-exponential factor, $E_{a}^{C}$ is the activation energy, $R$ is the gas constant, $T$ is

147 the absolute ambient temperature, $A_{C}$ is Arrhenius correction factor. The fitted curve is shown as the 
148 dashed line in Fig. 4, which is close to the measured capacity points.

In order to find out how the battery resistance changes at different temperatures and SOCs, the Hybrid Pulse Power Characteristic (HPPC) [16] test is conducted, where the resistance refers to the total battery resistance. Firstly, a fully charged battery is put in the incubator of $40^{\circ} \mathrm{C}$ and discharged with constant current of $1 / 3 \mathrm{C}$ to the SOC of $90 \%, 70 \%, 50 \%, 30 \%$ and $10 \%$ successively, and the incubator temperature to $30^{\circ} \mathrm{C}, 20^{\circ} \mathrm{C}, 10^{\circ} \mathrm{C}, 0^{\circ} \mathrm{C},-10^{\circ} \mathrm{C}$ and $-20^{\circ} \mathrm{C}$, repeat the above steps, and measure the battery resistance.

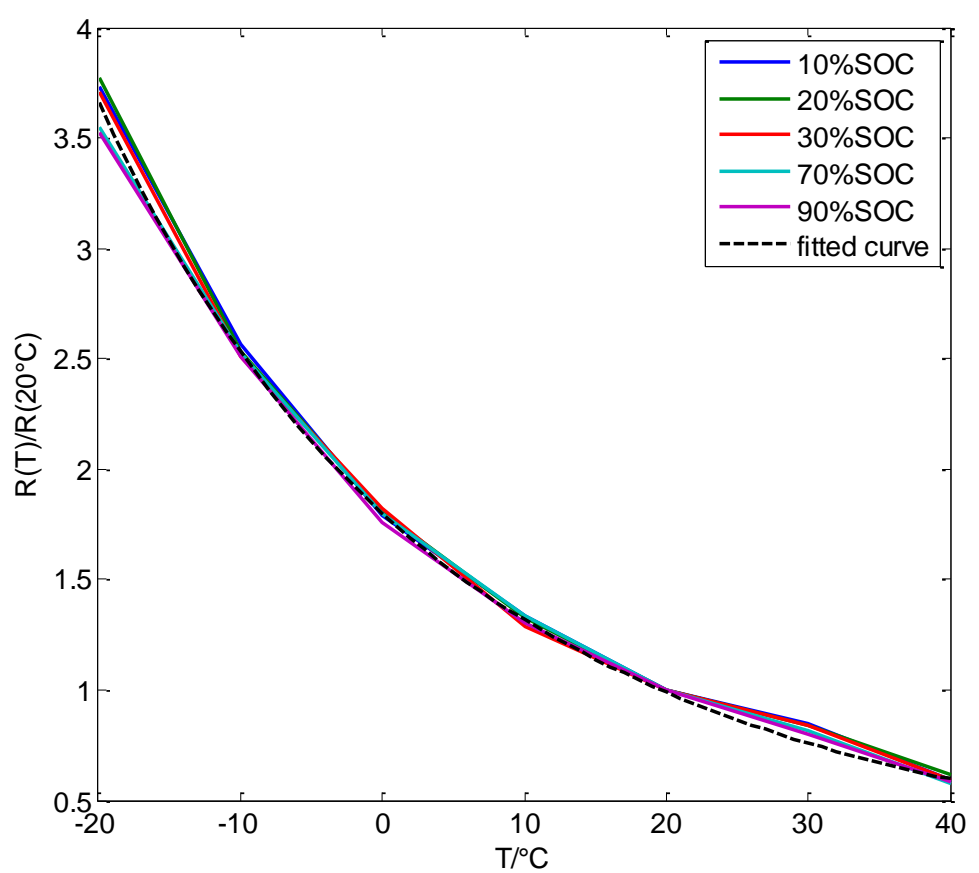



the ambient temperature. The dashed line is the fitted curve. The figure shows that the resistance

164 changes fast at low ambient temperatures. Furthermore, the resistances at different SOCs (higher than $10 \%$ ) remain almost the same when temperature is fixed. Therefore, the impact of SOC on resistance can be ignored when the battery SOC is higher than $10 \%$. As shown in Ref. [11], the resistance of the battery changes fast when SOC is lower than $10 \%$, so the effect of SOC on resistance cannot be ignored when SOC is lower than $10 \%$. effect of temperature on the LIB resistance, where $R_{T}$ is the resistance at temperature $T, R_{0}$ is the resistance at $20^{\circ} \mathrm{C}$. The curve is fitted by Arrhenius equation, and $\lambda_{R}$ can be expressed as:

$$
\lambda_{R}=R_{T} / R_{0}=B_{R} e^{-\mathrm{E}_{\mathrm{a}}^{R} / R T}+A_{R}
$$

where $B_{R}$ is the pre-exponential factor, $E_{a}^{R}$ is the activation energy, $R$ is the gas constant, $T$ is the absolute ambient temperature, $A_{R}$ is Arrhenius correction factor. The fitted curve is shown as the dashed line in Fig.5, which is close to the measured resistance points. used battery models are electrochemical model [17], equivalent circuit model $[18,19]$ and neural network model [20]. The electrochemical reaction mechanism of LIB is very complex. Therefore, the simplified electrochemical model such as the Shepherd, Unnewehr, Nernst model is used to describe the battery characteristics. Equivalent circuit model in $[18,19]$ describes the LIB from external electrical characteristics and PNGV, Thevenin and Rint model are typical equivalent circuit models. The calculation of neural network model in [20] is too complicated, and the estimation error 
will be affected by the training data and training methods. Thus the combined electrochemical model $(\mathrm{CM})$ from $[1,21,22]$ is used in this paper. This CM consists of the Shepherd, Unnewehr and Nernst model, which solves the problem that the three models only match partially the LIB voltage characteristic: For example, the Shepherd model only considers the end of battery charge and discharge, and the Unnewehr model cannot express the voltage platform. Thus the CM model can provide better fitting in the whole charge and discharge interval. However, the effect of temperature on battery parameters is ignored which will lead to the poor accuracy at low temperatures. Therefore, the above temperature models are merged to this combined electrochemical model to describe the dynamic behavior of LIB at various temperatures. Details are given below:

$$
\begin{aligned}
& \operatorname{SOC}(t)=\operatorname{SOC}(0)-\int_{0}^{\mathrm{t}} \eta i(t) \mathrm{d} t /\left(\lambda_{C} C_{0}\right) \\
& \begin{aligned}
E(t)= & E_{0}-\lambda_{R} R_{0} i(t)-K_{0} / \operatorname{SOC}(t)-K_{1} \operatorname{SOC}(t) \\
& +K_{2} \ln (\operatorname{SOC}(t))+K_{3} \ln (1-\operatorname{SOC}(t))
\end{aligned}
\end{aligned}
$$

where $\operatorname{SOC}(t)$ is the instantaneous $\mathrm{SOC}$ at time $t, \operatorname{SOC}(0)$ is the initial $\mathrm{SOC}, \eta$ is the charging/discharging efficiency, $i(t)$ is the current at time $t, C_{0}$ is the capacity at room temperature, $E(t)$ is the terminal voltage at time $t, E_{0}$ is the OCV of the fully charged battery, $R_{0}$ is the resistance at room temperature, $K_{0}, K_{1}, K_{2}$ and $K_{3}$ are constants. $\lambda_{C}$ and $\lambda_{R}$ are capacity and resistance temperature compensation coefficient respectively.

\section{SOP estimation considering battery temperature}

\subsection{SOC estimation method}

Extended Kalman Filter (EKF) is a popular algorithm to solve the nonlinear system state estimation problem [22]. Now Eq. (3) and Eq. (4) are discretized to obtain the state-space model of LIB. Let $x_{k}=\mathrm{SOC}_{k}, \quad y_{k}=E_{k}, \quad u_{k}=i_{k}$, then: 


$$
\begin{aligned}
& y_{k}=g\left(x_{k}, u_{k}\right)+v_{k}=E_{0}-\lambda_{R} R_{0} u_{k}-K_{0} / x_{k} \\
& -K_{1} x_{k}+K_{2} \ln \left(x_{k}\right)+K_{3} \ln \left(1-x_{k}\right)+v_{k}
\end{aligned}
$$

Linearizing Eq. (6) and Eq. (7), and we will obtain:

$$
\left\{\begin{array}{l}
x_{k}=A x_{k-1}+B u_{k-1}+w_{k} \\
y_{k}=C x_{k}+D u_{k}+E+v_{k}
\end{array}\right.
$$

$210 \quad$ where $A=\frac{\partial f\left(x_{k-1}, u_{k-1}\right)}{\partial x_{k-1}} \mid x_{k-1}=\hat{x}_{k-1}=1$,

$$
B=-\eta \Delta t /\left(\lambda_{C} C_{0}\right),
$$

$$
C=\frac{\partial g\left(x_{k}, u_{k}\right)}{\partial x_{k}} \mid x_{k}=\hat{x}_{k}=\frac{K_{0}}{\left(\hat{x}_{\bar{k}}\right)^{2}}-K_{1}+\frac{K_{2}}{\hat{x}_{\bar{k}}}-\frac{K_{3}}{1-\hat{x}_{\bar{k}}},
$$

$$
D=-\lambda_{R} R_{0},
$$

In EKF estimation method, $\mathrm{SOC}$ is calculated with $\mathrm{AH}$ integral method, and put into the observation equation to calculate the Kalman gain of each step. It reflects the residuals on the state variable weights of SOC, and then the optimal SOC estimation is obtained from the updated state estimation equation.

\subsection{SOP estimation with AMPC}

In order to ensure the accuracy of the SOP estimation, the AMPC is combined with EKF to estimate the SOP of LIB. Within a short time duration $\Delta t$, the battery can be assumed to be discharged at a constant current, and after the short time $\Delta t$ about 10 seconds [13,24], $\operatorname{SOC}(t+\Delta t)=\operatorname{SOC}_{\min }$ or $V(t+\Delta t)=V_{\min }$. Then the discharge peak power is

$$
\operatorname{SOP}(t)=i_{\max }(t) E\left(t, i_{\max }(t)\right)
$$


226 where $\operatorname{SOP}(t)$ is the instantaneous state of charge at time $t, i_{\max }(t)$ is the estimated peak current at 227 time $t, E\left(t, i_{\max }(t)\right)$ is the terminal voltage at time $\mathrm{t}$ when the current is $i_{\max }(t)$.

228 Based on the above models, the peak current can be calculated from SOC limit, which is 229 denoted by $u_{\max }^{\mathrm{SOC}}$ and equals:

$$
u_{\max }^{\mathrm{SOC}}=B^{-1}\left(x_{k}-x_{\min }\right)
$$

This peak current can also be calculated from voltage limit, which is represented by $u_{\max }^{\text {volt }}$ and 232 equals:

$$
u_{\max }^{\mathrm{volt}}=D^{-1}\left(y_{\min }-C x_{k}-E\right)
$$

Note also that manufacturers also provide the rated battery maximum current, which is denoted by $u_{\max }$, therefore, these notations can be combined together and the following maximum allowed peak discharge current $u_{\max }^{\text {dis }}$ is defined as

$$
u_{\max }^{\mathrm{dis}}=\min \left(u_{\max }, u_{\max }^{\mathrm{SOC}}, u_{\max }^{\mathrm{volt}}\right)
$$

Therefore, the maximum allowed discharge peak power is

$$
\mathrm{SOP}_{\text {max }}^{\mathrm{dis}}=u_{\max }^{\mathrm{dis}} y\left(k, u_{\text {max }}^{\mathrm{dis}}\right)
$$

The detail SOC and SOP estimation process is shown as follows: 


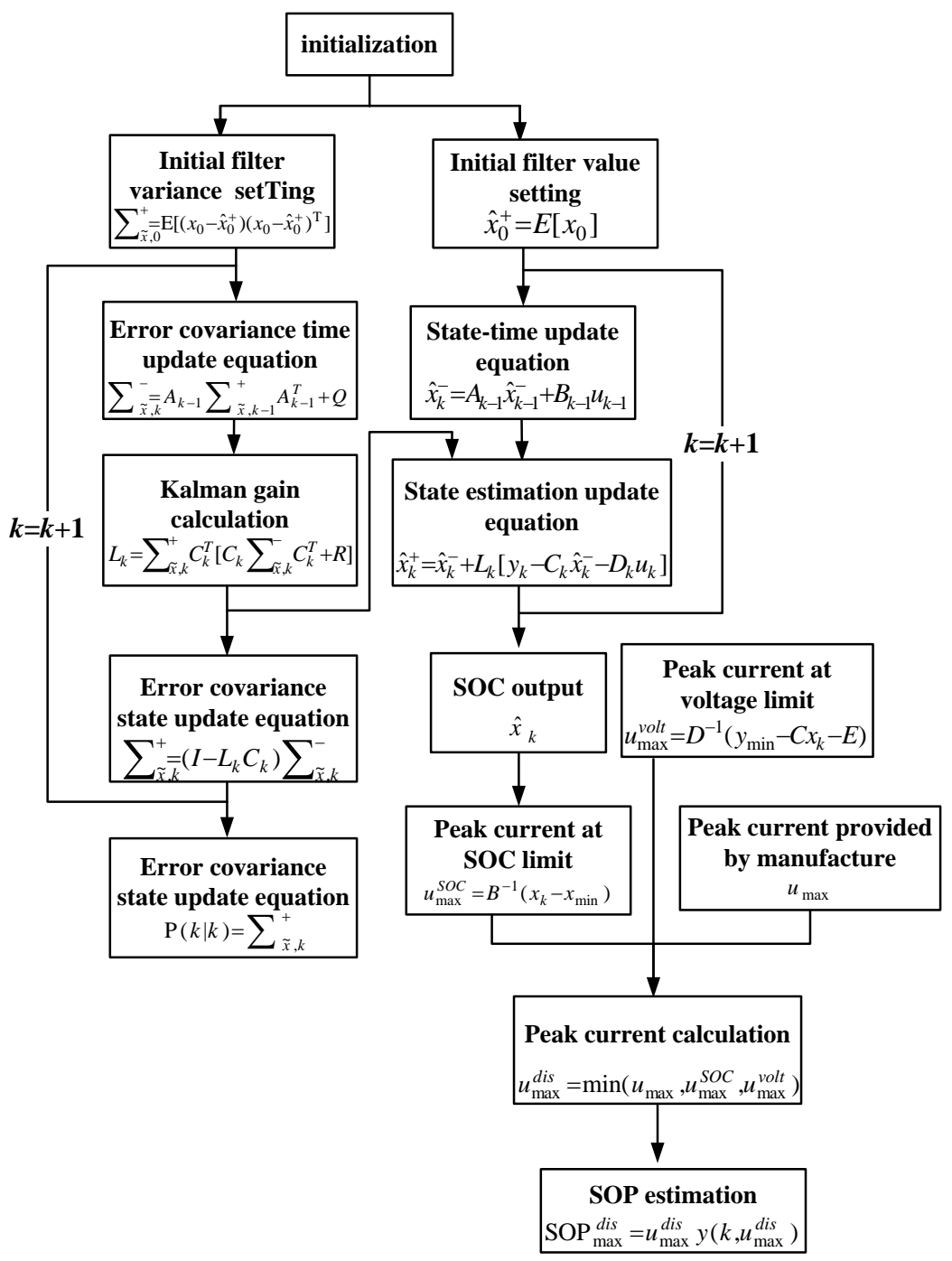

Fig.6 SOP estimation flow chart

Form the above flow chart, we can see that the algorithm is very simple and straightforward,

244 and it is suitable for practical engineering applications such as Battery Management System (BMS).

245 In order to validate the accuracy of the estimation algorithm, the simulations and experiments are

246 conducted in Section 4.

\section{Simulations and Experiments}

\subsection{Parameters identification}

In order to identify the model parameters, we choose the 9Ah Lithium iron phosphate battery 
and conduct the constant current discharge test and HPPC test. The measured data are fitted by

251 Arrhenius equation to obtain the temperature models of capacity and resistance as shown in Fig.4 252 and Fig.5. The model parameters are identified by the least squares method, and the results of 253 parameters for the temperature models of battery capacity and residence are shown in Table 2 and 254 Table 3:

Table 2 Parameters for the temperature model of battery capacity

\begin{tabular}{cccc}
\hline Parameter & $B_{C}$ & $E_{\mathrm{a}}^{C}$ & $A_{C}$ \\
\hline Value & $-1.683 * 10^{-6}$ & $-2.77 * 10^{4}$ & 1.117 \\
\hline
\end{tabular}

Table 3 Parameters for the temperature model of battery residence

\begin{tabular}{cccc}
\hline Parameter & $B_{R}$ & $E_{\mathrm{a}}^{R}$ & $A_{R}$ \\
\hline Value & $1.861 * 10^{-4}$ & $-2.08 * 10^{4}$ & $5.23 * 10^{-2}$ \\
\hline
\end{tabular}

\subsection{The Dynamic Battery Model Validation}

In order to validate the dynamic model in Eq. (4), a single $\mathrm{LiFePO}_{4}$ cell with a nominal capacity 261 of $9 \mathrm{Ah}$ is chosen to be tested in an incubator with the temperature of $-10^{\circ} \mathrm{C}, 0^{\circ} \mathrm{C}$ and $25^{\circ} \mathrm{C}$ 262 successively. The battery is fully charged and the DST curve is chosen as the discharge curve which 263 is provided in "USABC Electric vehicle battery test procedures manual" [13]. And the comparison 264 between the voltage response curve of the dynamic model, the voltage response of the initial model 265 and the test voltage is shown as follows. 


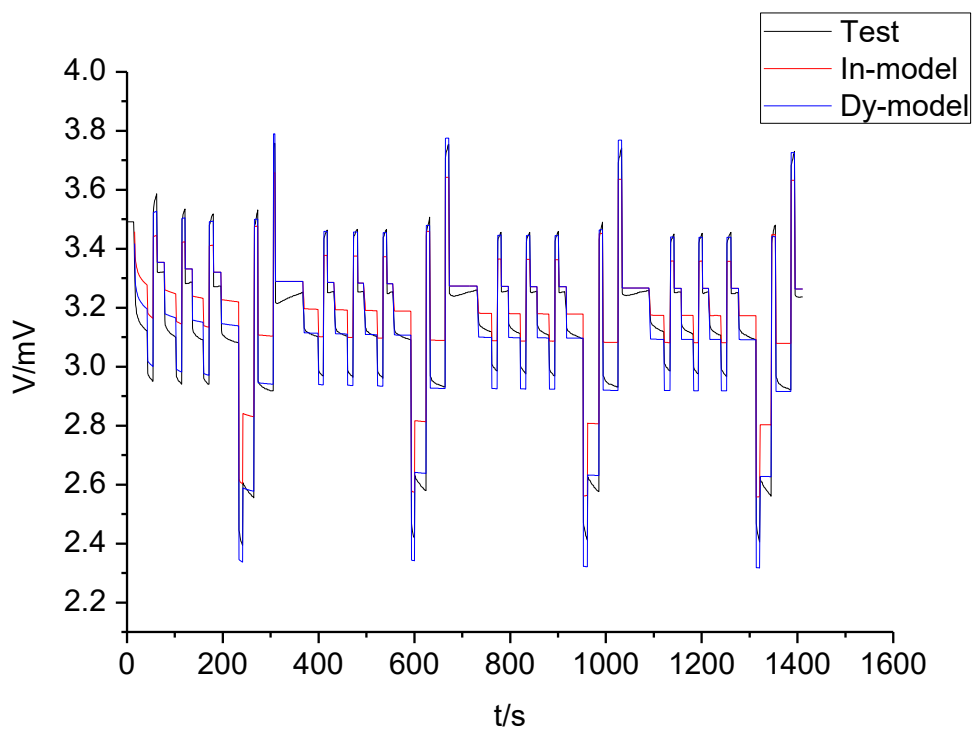

Fig.7 The validation of dynamic model

In Fig.7 the voltage response curve of the dynamic model (Dy-model), the voltage response of 269 the initial model (In-model) and the test voltage (Test) are shown respectively. The proposed 270 dynamic battery model is obviously better than the initial model.

\section{$271 \quad 4.3$ SOC Estimation Validation}

The single $\mathrm{LiFePO}_{4}$ cell with a nominal capacity of $9 \mathrm{Ah}$ is chosen to be tested in an incubator

273 with the temperature of $-10^{\circ} \mathrm{C}, 0^{\circ} \mathrm{C}$ and $25^{\circ} \mathrm{C}$ successively. The battery is fully charged and the DST

274 curve is chosen as the discharge curve which is provided in "USABC Electric vehicle battery test 275 procedures manual" [13]. The SOC at fixed room temperature (SOC at FRT), the SOC at fixed 276 ambient temperature (SOC at FAT), the SOC at dynamic battery temperature (SOC at DBT) and the 277 real SOC are shown as follows: 


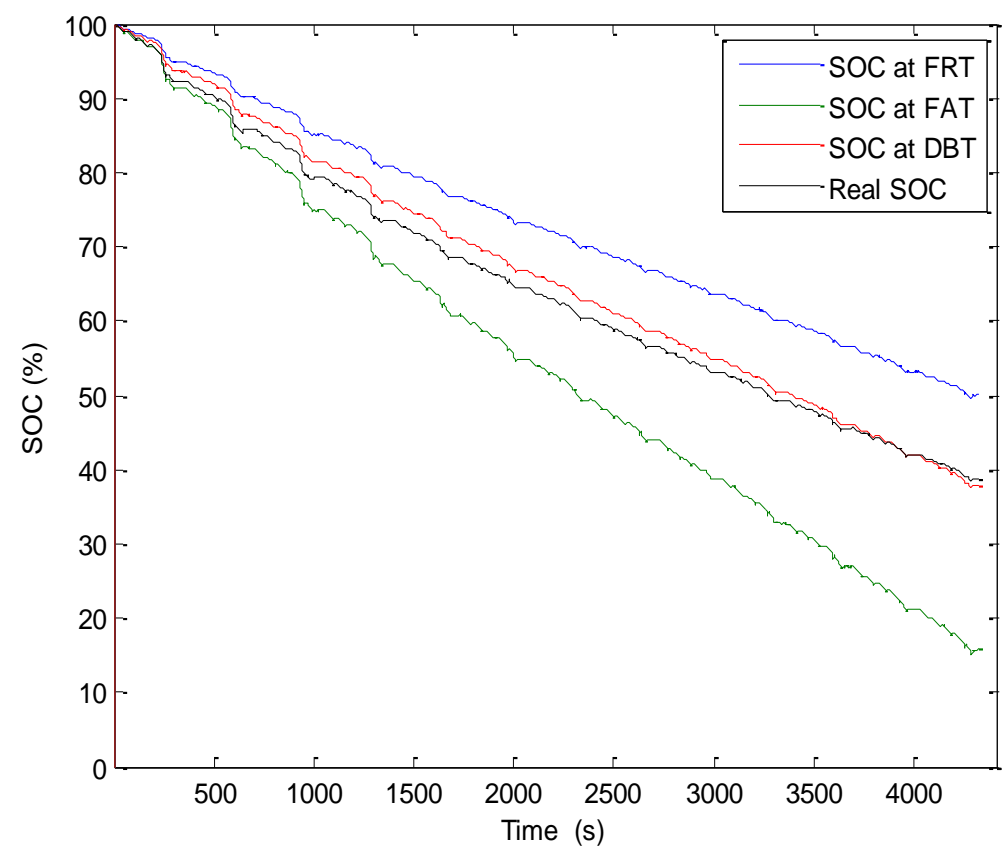

Fig.8 SOC estimation result at $-10^{\circ} \mathrm{C}$

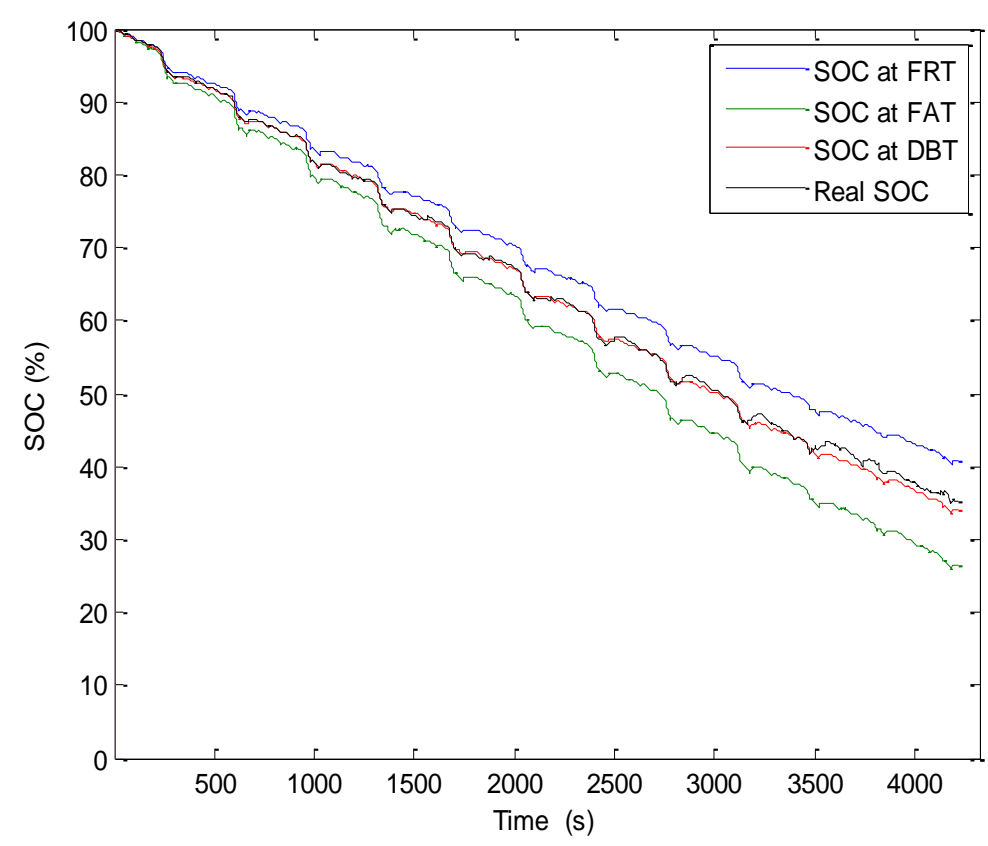

Fig.9 SOC estimation result at $0^{\circ} \mathrm{C}$ 


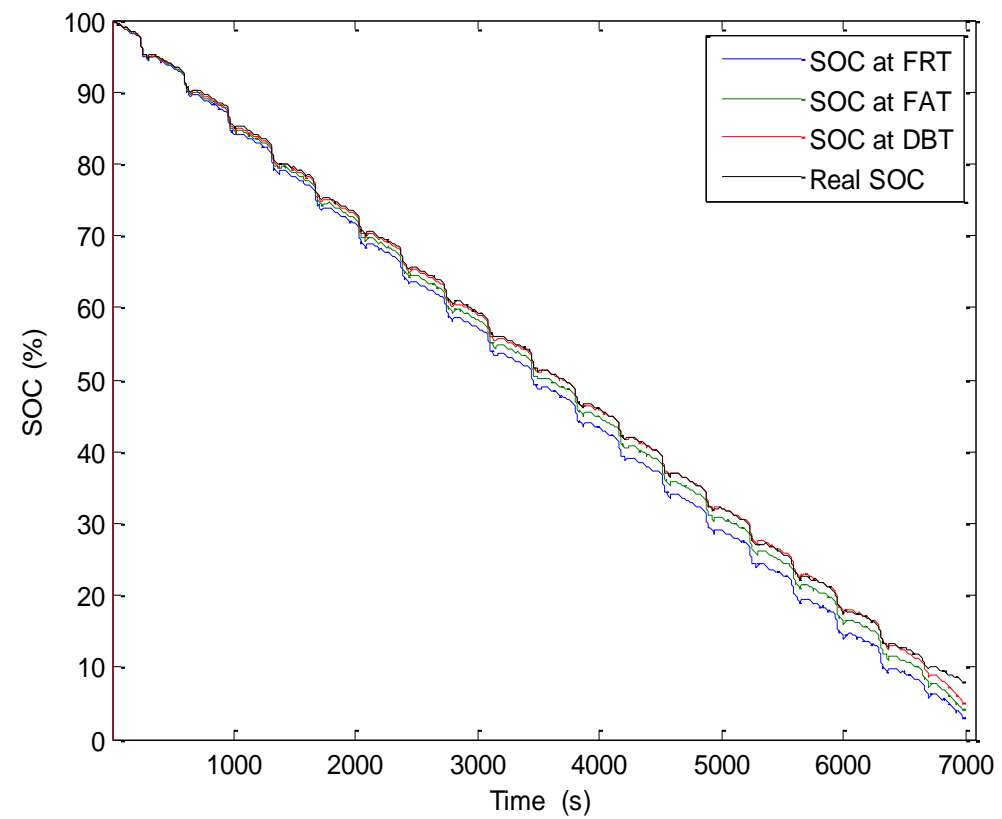

Fig. 10 SOC estimation result at $25^{\circ} \mathrm{C}$

In Fig.8, Fig.9 and Fig.10, the SOC estimation curves after DST at temperature of $-10^{\circ} \mathrm{C}, 0^{\circ} \mathrm{C}$ and $25^{\circ} \mathrm{C}$ are shown respectively. And the maximum errors are shown in Table 4:

Table 4 Maxmum Errors of SOC Estimation at FRT, FAT and DBT

\begin{tabular}{cccc}
\hline Ambient Temperature & Max-error of FRT & Max-error of FAT & Max-error of DBT \\
\hline$-10^{\circ} \mathrm{C}$ & $11.89 \%$ & $22.40 \%$ & $3.66 \%$ \\
\hline $0^{\circ} \mathrm{C}$ & $5.92 \%$ & $9.61 \%$ & $2.07 \%$ \\
\hline $25^{\circ} \mathrm{C}$ & $4.91 \%$ & $3.93 \%$ & $2.94 \%$ \\
\hline
\end{tabular}

From Table 4, we can see that the error of SOC at DBT is smaller than SOC at FRT and SOC at

289 FAT, especially at low temperature. The reason is that the resistance of LIB is higher at low 290 temperatures, so the battery temperature rises higher at DST. Besides, same temperature variation 291 has a bigger impact on the parameters at low temperature as shown in Fig. 4 and Fig. 5. These 292 reasons cause a poor performance of SOC estimation methods which do not consider temperature 293 impact. 


\subsection{SOP Estimation Validation}

In order to verify the SOP estimation algorithm, the real SOP of the battery at different

297 temperature needs to be tested. The test method recommended in [24] is adopted in this paper. The 298 battery is discharged and charged alternatively with the current of $1 \mathrm{C}, 2 \mathrm{C}, 5 \mathrm{C}$ and $10 \mathrm{C}$ at certain 299 SOC and temperature shown in Fig.2 and Fig. 3. In this process, the voltage of the battery is 300 recorded and fitted to obtain the maximum current at the cut-off voltage. Then the peak power of the 301 battery at any SOC and temperature point is the multiplication of the cut-off voltage and maximum 302 current. The SOP at fixed room temperature (SOP at FRT), the SOP at fixed ambient temperature 303 (SOP at FAT), the SOP at dynamic battery temperature (SOP at DBT) and the real SOP are shown as 304 follows:

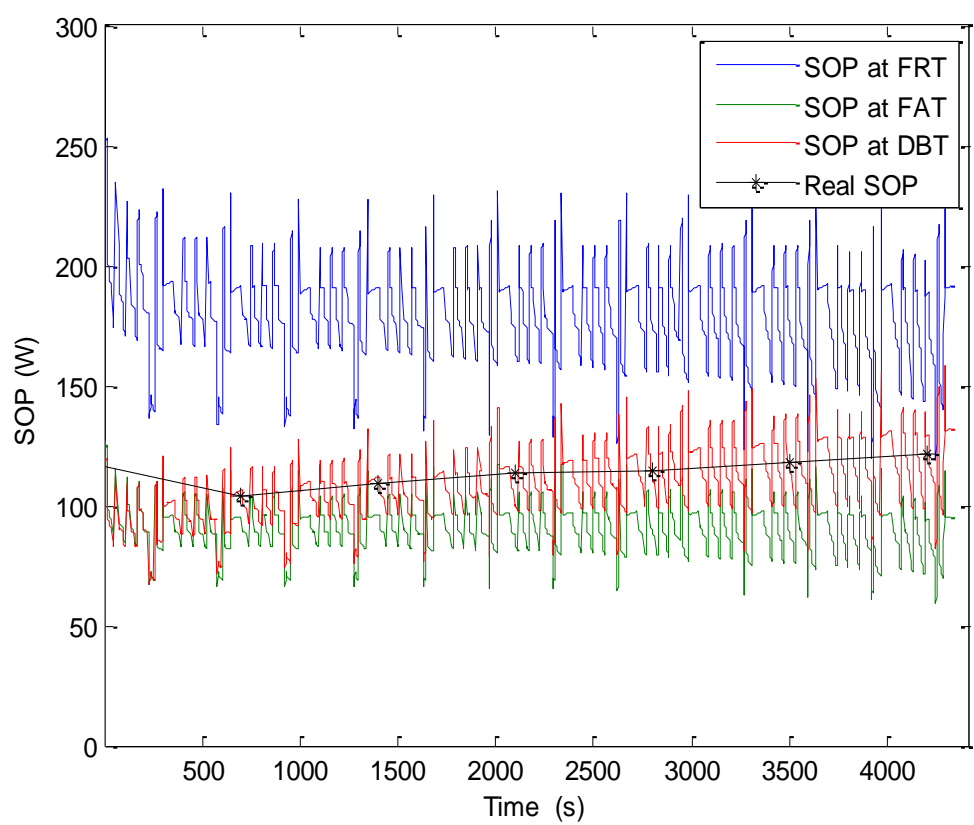




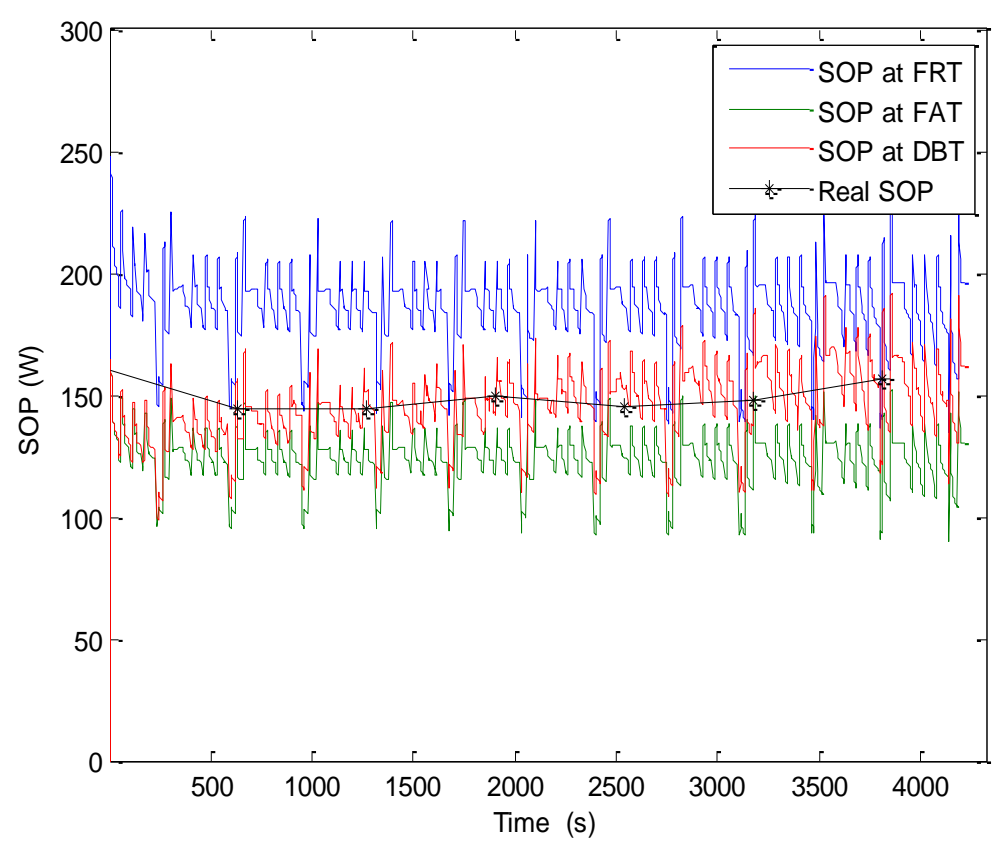

Fig. 12 SOP estimation result at $0^{\circ} \mathrm{C}$

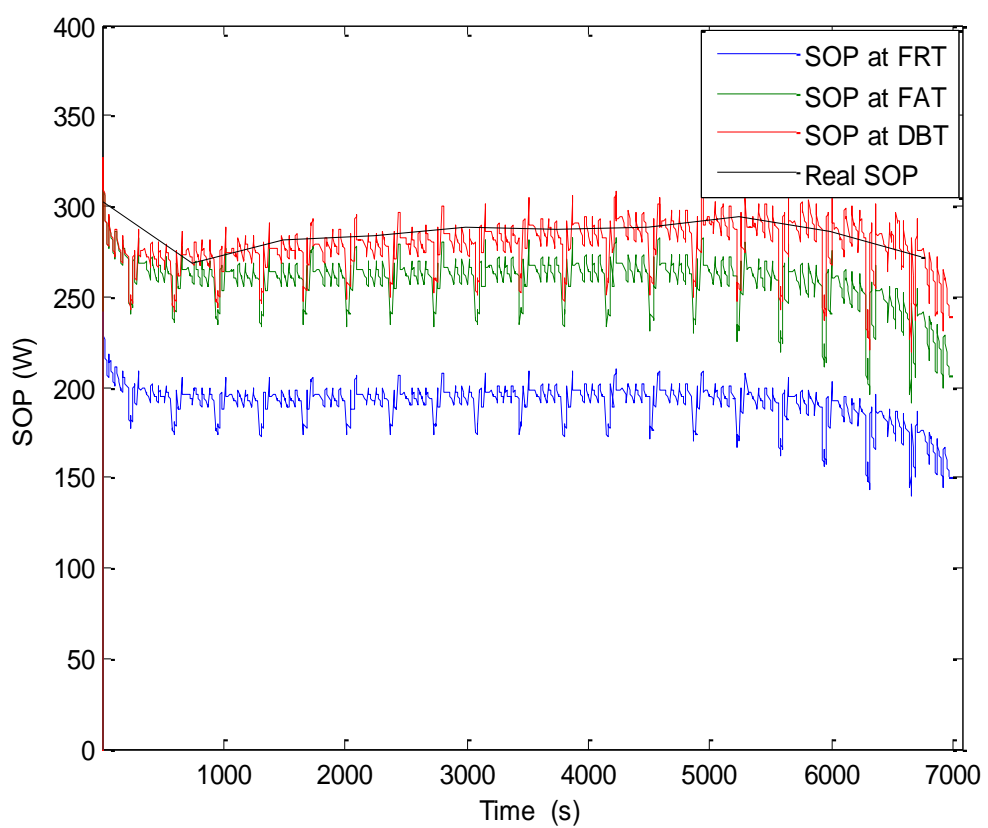

Fig.13 SOP estimation result at $25^{\circ} \mathrm{C}$

In Fig.11, Fig.12 and Fig.13, the SOP estimation curves after DST and the real SOP at

313 temperature of $-10^{\circ} \mathrm{C}, 0^{\circ} \mathrm{C}$ and $25^{\circ} \mathrm{C}$ are shown respectively. In these figures, SOP at DBT which is 314 nearer to the real SOP curve performs better than SOP at FRT and SOP at FAT. Comparing the three 
315 SOP curves, we will see that the result of SOP at FRT is higher than the results of SOP at FAT and 316 DBT at low temperatures; but it is lower at high temperatures. The reason is that the battery 317 resistance is higher at low temperatures and lower at high temperatures. When comparing SOP at 318 FAT and DBT, the result of DBT is nearly the same with the result of FAT at the initial discharging 319 process. The results of SOP at DBT increase quicker than SOP at FAT because the battery capacity 320 increases and resistance decreases due to the rising temperature. Thus, the SOP at FRT will lead to 321 higher peak power which exceeds the one withstood by the battery at lower temperatures, and the 322 SOP at FAT will lead to lower peak power which cannot ensure effectively battery performance. When compared to the HPPC method and the method based on SOC limits (MSL) at dynamic 324 battery temperatures, the following Fig. 14 is obtained.

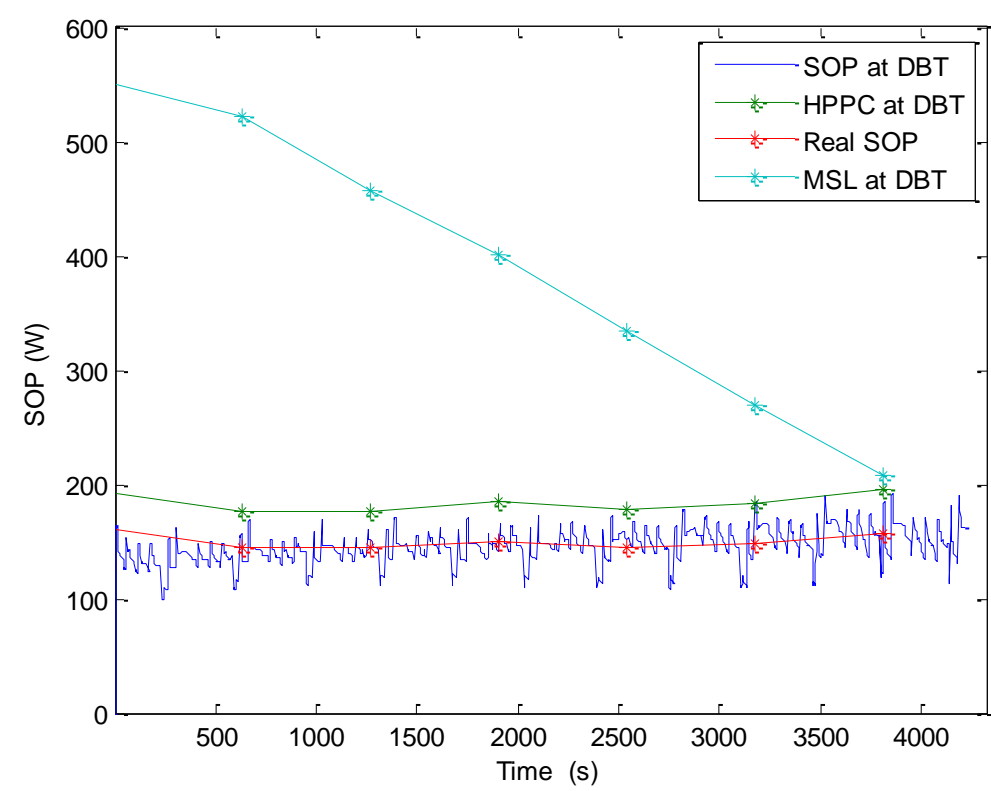

Fig.14 SOP estimation result at $0^{\circ} \mathrm{C}$

In Fig.14, the SOP estimation curves with different methods after DST and the real SOP at temperature $0^{\circ} \mathrm{C}$ are shown respectively. As analyzed in Section 1, the SOP with HPPC and MSL method are high than the proposed methods (SOP at DBT). Especially, the results of MSL methods 
330 is much high than the Real SOP.

Analyzing the SOP estimation results at different temperature further in Fig. 15, it is found that

332 the peak discharge currents are around $3.62 \mathrm{C}, 5.42 \mathrm{C}$ and $10.13 \mathrm{C}$ at temperature of $-10^{\circ} \mathrm{C}, 0^{\circ} \mathrm{C}$ and $33325^{\circ} \mathrm{C}$ respectively, which means that the LIB is unable to achieve a high discharging rate and cannot 334 meet the demands of starting or acceleration of the electric vehicle at low temperature. Thus, heating 335 equipment and thermal insulations must be added to the LIB pack to meet the driving demand at low 336 temperatures.

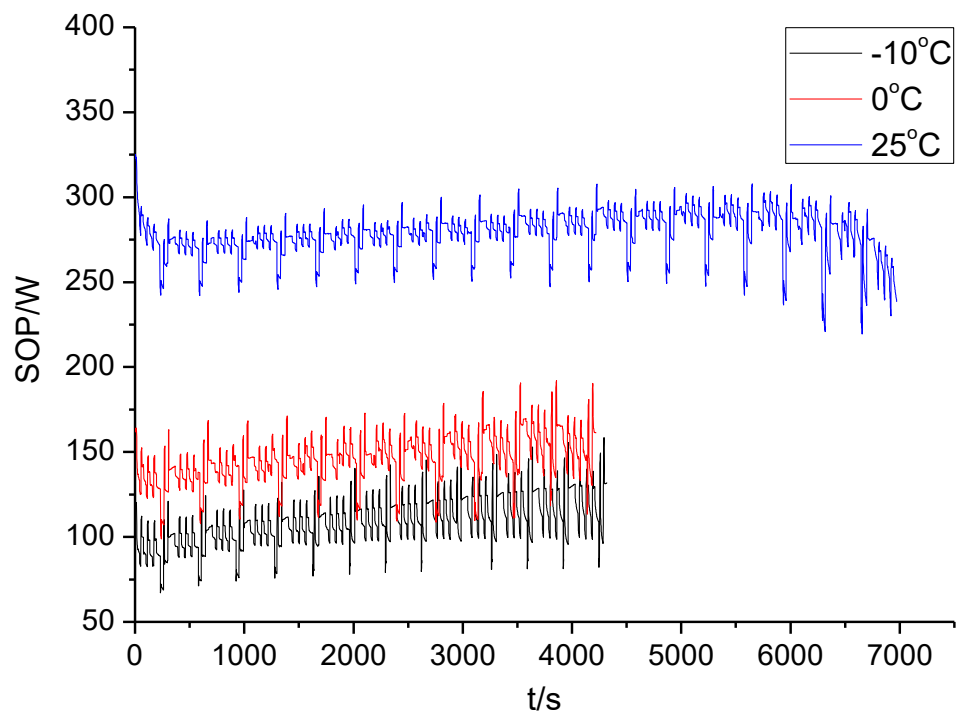

The battery SOP, SOC and terminal voltage are compared at the discharge platform of the LIB 340 and the end of discharge at $25^{\circ} \mathrm{C}$ in Fig. 14 and Fig. 15. The SOC intervals are $77.23 \% \sim 85.41 \%$ and $341 \quad 5.12 \% \sim 12.03 \%$ respectively. 


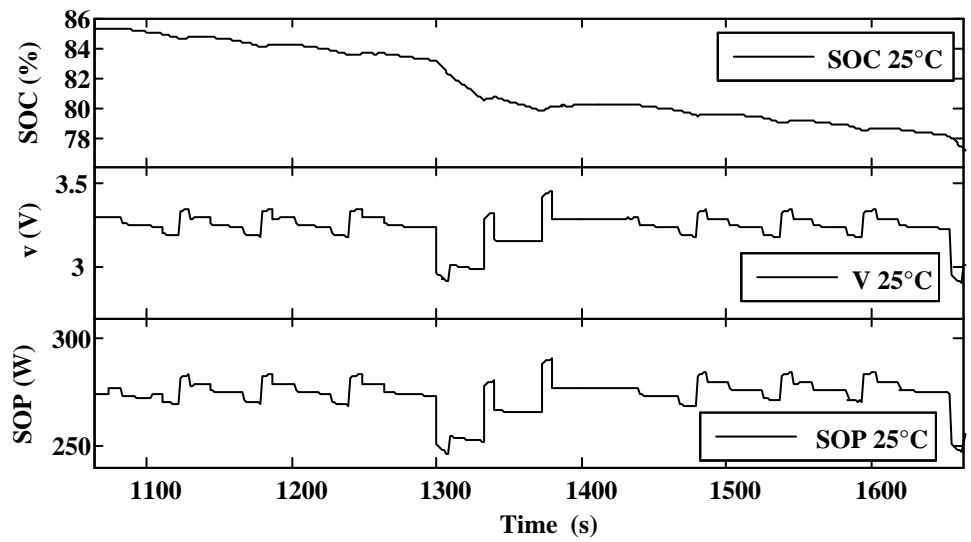

Fig.16 Comparison of SOC, terminal voltage and SOP of LIB at voltage platform

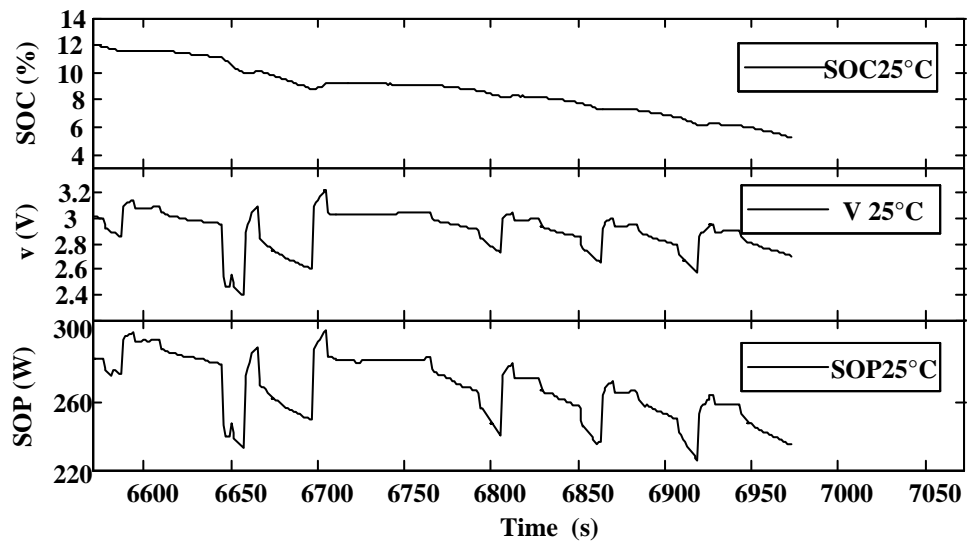

Fig.17 Comparison of SOC, terminal voltage and SOP of LIB at the end of discharge

As shown in Fig.16 and Fig.17, the SOP changing trend is nearly the same with the terminal

348 terminal voltage can reflect the peak power to certain extent. However, at the end of the discharge,

349 the SOP decreases faster than the battery terminal voltage due to a rapid increase in battery

350 resistance where the SOC is lower than $10 \%$. So the battery will have to withstand a greater impulse

351 current if only the terminal voltage is used as the control parameter, and this might damage the system security and battery life. 
At low temperatures, key LIB characteristic parameters, such as battery capacity and resistance, change significantly when the ambient temperature is low. In this paper, a new SOP estimation method is developed, which includes the establishment of battery temperature, capacity and resistance based on experimental data, and also a combination of the AMPC method and the EKF technique to study the impact of temperature to SOP. The following conclusions can be obtained after analyzing the SOP estimation results:

1) The newly proposed SOP estimation algorithm performs better than the algorithm at fixed room temperature and the algorithm at fixed ambient temperature. And also the proposed method 362 shows high accuracy when compared to the existing method at dynamic battery temperatures.

2) The SOP curve shows the same trend with the terminal voltage of the discharge platform, 364 and decreases faster at the end of discharge. Thus, system security and battery life will be damaged 365 if the terminal voltage at the end of discharge is used as the only control parameter. voltage platform is only $3.62 \mathrm{C}$ at the temperature of $-10^{\circ} \mathrm{C}$ and cannot meet the demands of vehicle starting and acceleration. Thus, heating equipment and thermal insulations must be added to the LIB 369 pack at low temperature.

\section{Acknowledgments}

This work is supported by the National Natural Science Foundation of China (21373074) and the International Cooperation Projects of Anhui Province in China (1303063010). 


\section{Reference}

[1] G.L. Plett, High-performance battery-pack power estimation using a dynamic cell model, IEEE Transactions on Vehicular Technology, 2004, 53: 1586-1593.

[2] F. Sun, R. Xiong, H. He, et al. Model-based dynamic multi-parameter method for peak power estimation of lithium-ion batteries, Applied Energy, 2013, 96: 378-386.

[3] R. Xiong, H. He, F. Sun, et al. Model-based state of charge and peak power capability joint estimation of lithium-ion battery in plug-in hybrid electric vehicles. Journal of Power Sources, 2013, 229: 159-169.

[4] S. Yoon, I. Hwang, C. Lee, et al. Power capability analysis in lithium ion batteries using electrochemical impedance spectroscopy. Journal of Electroanalytical Chemistry, 2011, 655, $32-38$.

[5] X. Hu, R. Xiong, B. Egardt. Model-based dynamic power assessment of lithium-ion batteries considering different operating conditions[J]. IEEE Transactions on Industrial Informatics, 2014, 10(3): 1948-1959.

[6] C Burgos-Mellado, M E Orchard, M Kazerani, et al. Particle-filtering-based estimation of maximum available power state in Lithium-Ion batteries. Applied Energy, 2016, 161: 349-363.

[7] C. Fleischer, W. Waag, Z. Bai, et al. Self-learning state-of-available-power prediction for lithium-ion batteries in electrical vehicles. IEEE Vehicle Power and Propulsion Conference (VPPC), 2012, 370-375.

[8] C. Zhang, C. Zhang, S.M. Sharkh. Estimation of real-time peak power capability of a traction battery pack used in an HEV. Power and Energy Engineering Conference (APPEEC), 2010, 1-6. 
396 [9] R. Xiong, H. He, F. Sun, K. Zhao, Online estimation of peak power capability of Li-ion batteries in electric vehicles by a hardware-in-loop approach, Energies 2012, 5: 1455-1469.

[10] M. Dubarry, C. Truchot C, B.Y. Liaw, et al. Evaluation of commercial lithium-ion cells based on composite positive electrode for plug-in hybrid electric vehicle applications III: effect of thermal excursions without prolonged thermal aging. Journal of the Electrochemical Society, 2013, 160(1): A191-A199.

[11]X. Liu, Z. Chen, C. Zhang, et al. A novel temperature-compensated model for power Li-ion 263-272.

[12]W. Waag, C. Fleischer, D.U. Sauer. Adaptive on-line prediction of the available power of lithium-ion batteries. Journal of Power Sources, 2013, 242: 548-559.

[13]SAND 99-0497, USABC. Electric vehicle battery test procedures manual. United States Advanced Battery Consortium, 1996.

[14]C. Zhu, X. Li, L. Song, et al. Development of a theoretically based thermal model for Lithium-ion battery pack. Journal of Power Sources, 2013, 223(1): 155-164.

[15]M. Uno, A. Kukita. Cycle Life Evaluation Based on Accelerated Aging Testing for Lithium-Ion Capacitors as Alternative to Rechargeable Batteries. IEEE Transactions on Industrial Electronics, 2016, 63(3): 1607-1617.

[16]DOE/ID-11069, FreedomCAR Battery Test Manual for Power-Assist Hybrid Electric Vehicles. U.S. Department of Energy, 2003.

[17]M. Corno, N. Bhatt, S.M. Savaresi, et al. Electrochemical model-based state of charge 
estimation for Li-ion cells. IEEE Transactions on Control Systems Technology, 2015, 23(1): $117-127$.

[18] Y. Wang, C. Zhang, Z. Chen. An adaptive remaining energy prediction approach for lithium-ion batteries in electric vehicles. Journal of Power Sources, 2016, 305: 80-88.

[19] S. Nejad, D.T. Gladwin, DA Stone. A systematic review of lumped-parameter equivalent circuit models for real-time estimation of lithium-ion battery states. Journal of Power Sources, 2016, 316: 183-196.

[20] M. Ibrahim, S. Jemei, G. Wimmer, et al. Nonlinear autoregressive neural network in an energy management strategy for battery/ultra-capacitor hybrid electrical vehicles. Electric Power Systems Research, 2016, 136: 262-269.

[21]Y. He, X. Liu, C. Zhang, et al. A new model for State-of-Charge (SOC) estimation for high-power Li-ion batteries. Applied Energy, 2013, 101: 808-814.

[22]L. Zhong, C. Zhang, Y. He, et al. A method for the estimation of the battery pack state of charge based on in-pack cells uniformity analysis. Applied Energy, 2014, 113: 558-564.

[23]H. He, H. Qin, X. Sun, et al. Comparison study on the battery SOC estimation with EKF and UKF algorithms. Energies, 2013, 6(10): 5088-5100.

[24] Japan Electric Vehicle Society. Test method of input and output power density of nickel-hydride battery for Hybrid Electric Vehicles. Japan: 2003. 